\title{
Aharonov-Bohm cages in the GaAlAs/GaAs system
}

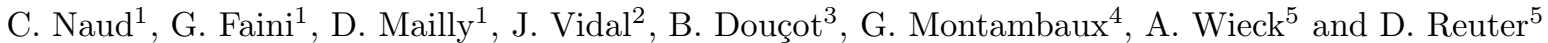 \\ ${ }^{1}$ Laboratoire de Photonique et de Nanostructures 196, avenue Henri Ravéra BP 29 F-92222 Bagneux cedex France \\ ${ }^{2}$ Groupe de Physique des Solides, CNRS UMR 7588, Universités Paris 6 et 7, \\ 2, place Jussieu, 75251 Paris Cedex 05 France \\ ${ }^{3}$ Laboratoire de Physique Théorique et Hautes Énergies, CNRS UMR 7589, Universités Paris 6 et 7 , \\ 4, place Jussieu, 75252 Paris Cedex 05 France \\ ${ }^{4}$ Laboratoire de Physique des Solides, CNRS UMR 8502, Université Paris Sud, Bât. 510, 91405 Orsay, France \\ ${ }^{5}$ Lehrstuhl für Angewandte Festkoerperphysik Ruhr-Universität Bochum \\ NB 03 / 58 Universitätsstrasse 150 D-44780 Bochum Germany
}

\begin{abstract}
Aharonov-Bohm oscillations have been observed in a lattice formed by a two dimensional rhombus tiling. This observation is in good agreement with a recent theoretical calculation of the energy spectrum of this so called $\mathcal{T}_{3}$ lattice. We have investigated the low temperature magnetotransport of the $\mathcal{T}_{3}$ lattice realized in the GaAlAs/GaAs system. Using an additional electrostatic gate, we have studied the influence of the channel number on the oscillations amplitude. Finally, the role of the disorder on the strength of the localisation is theoretically discussed.
\end{abstract}

PACS numbers : 73.23, 73.6,3 73.61.E, 73.20, 73.20.D

\section{INTRODUCTION}

The spectral properties of an electron in a periodic lattice in the presence of a magnetic field are of particular interest. Indeed, the frustration between the spatial periodicity and the one induced by the magnetic field gives rise to spectacular energy spectra versus the reduced flux $f=\phi / \phi_{0}$, where $\phi$ is the magnetic flux per elementary cell and $\phi_{0}$ is the flux quantum. A well-known example is the Hofstadter butterfly [1]. Recently, the study of a bipartite two-dimensional rhombus tiling, named $\mathcal{T}_{3}$, (shown in figure (11)) has attracted a lot of attention [2].

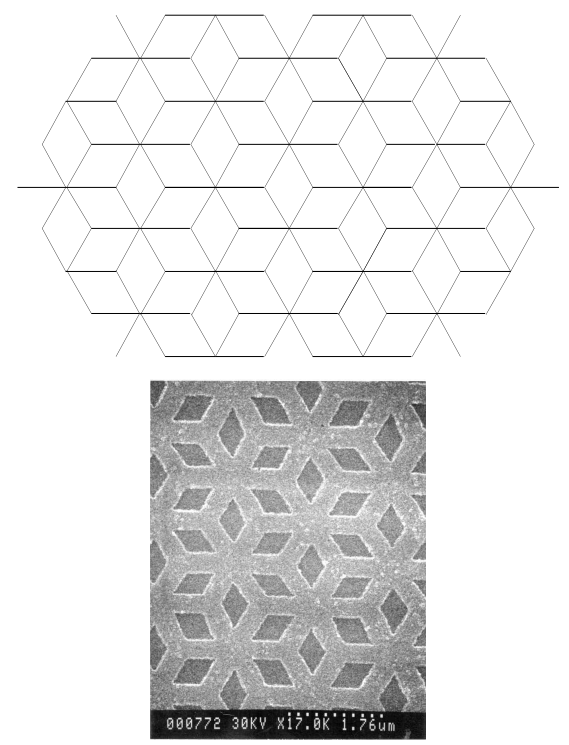

FIG. 1. top : A piece of the $\mathcal{T}_{3}$ lattice. bottom : Electron microscope view of the aluminium mask used to etch the 2DEG. The nominal width of the wires defining the network is $0.4 \mu \mathrm{m}$ whereas their length is about $1 \mu \mathrm{m}$.
For $f=1 / 2$, its energy spectrum is reduced to three degenerate discrete levels (see figure (2)). The whole lattice is then similar to a unique super-atom with localized electrons. This behavior is very different from the square lattice one where for any rational value of the reduced flux the energy spectrum is continuous.

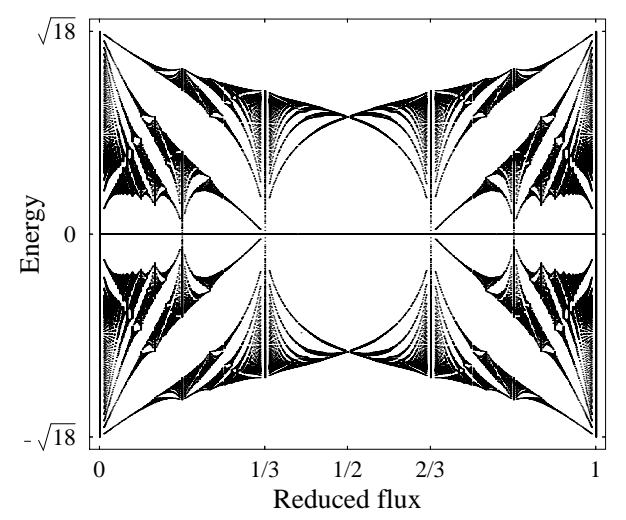

FIG. 2. Energy spectrum of the $\mathcal{T}_{3}$ lattice versus reduced flux.

This localization for the $\mathcal{T}_{3}$ lattice can be easily understood in terms of Aharonov-Bohm (AB) effect. Indeed, in order to propagate through the lattice, an electron must travel through $\mathrm{AB}$ loops. For $f=1 / 2$ interferences are destructive and the electron is then confined in a so-called AB cage. This tight-binding calculation has received an experimental confirmation in the temperature dependence of the critical current of a superconducting network in the $\mathcal{T}_{3}$ geometry. In addition, the unusual nature of the state at half flux quantum has been revealed by a strong reduction of the critical current and by a very disordered vortex pattern through decoration experiments [3: 
The first experimental evidence of $\mathrm{AB}$ cages in a normal metal network tailored in a high mobility two dimensionnal electron gas (2DEG) has recently been reported [5]. The low temperature magnetoresistance measurements show clear $h / e$ oscillations in arrays of 2500 cells. Experiments performed on square lattices of similar size do not show such a behavior. The temperature dependence of the $h / e$ peak amplitude of the Fourier transform for both the $\mathcal{T}_{3}$ lattice and the single rhombus have been compared. For a single loop the amplitude of the AB signal is expected to follow a $T^{-1 / 2}$ law as long as the size of the loop $L$ is smaller than the phase coherence length $L_{\Phi}$, because of temperature averaging. When the temperature is such that $L>L_{\Phi}$ the AB signal falls down exponentially [6]. Thus, the size of a cage being larger than that of a single loop, one expects a cutoff temperature significantly smaller for the $\mathcal{T}_{3}$ as compared to the one for a single rhombus. The experimental results show that this critical temperature is around $1 K$ for a rhombus whereas it is below $100 \mathrm{mK}$ for the $\mathcal{T}_{3}$. If one assumes a $T^{-1 / 3}$ temperature dependence for $L_{\Phi}$ in GaAlAs/GaAs 1D systems [7], the ratio of the measured critical lengths is 2.7 , in good agreement with the geometrical dimensions yielding a ratio of 3 . More strikingly, at high magnetic field, $h / 2 e$ oscillations appear whose amplitude can be much higher than the fundamental period. Such an amplitude dismisses a simple interpretation in terms of harmonics generation. Moreover, the temperature dependence of the $h / 2 e$ oscillations is similar to that of the $h / e$ one. This also indicates that the characteristic lengths associated with the two periodicities are alike and rules out any interpretation in terms of harmonics. One may also think of Aronov-Altshuler-Spivak (AAS) oscillations when dealing with an $h / 2 e$ signal. But, due to the aspect ratio of the sample, the AAS signal should vanish for high magnetic fields. Thus, any explanation in terms of AAS would imply that a high magnetic field induces the squeezing of the wires leading to an array of one dimensionnal leads. A second explanation for the frequency doubling could be the electron-electron interactions which could induce charge doubling. Up to now the origin of this $h / 2 e$ peak at high magnetic fields remains unclear.

In the present paper we report on new experiments on the cage effect. These experiments in an other type of sample confirm both the existence of the cage effects and the systematic presence of the frequency doubling for high magnetic fields. We have realised $\mathcal{T}_{3}$ lattices on a GaAlAs/GaAs heterostructure on which electrostatics gates have been deposited in order to control the number of channels per wire. The fabrication process and the experimental results concerning these devices are introduced in section 2. Finally, in section 3 we present theoretical calculations concerning the influence of the disorder on the cage effect.

\section{CAGE EFFECT VERSUS THE NUMBER OF CHANNELS}

This work is carried out on high-mobility 2DEG heterojunction material with an initial mobility of $3 \times 10^{6}$ $\mathrm{cm}^{2} \mathrm{~V}^{-1} \mathrm{~s}^{-1}$ and a carrier density of $3.7 \times 10^{11} \mathrm{~cm}^{-2}$ which give a Fermi wavelength $\lambda_{F}=41 \mathrm{~nm}$ and an elastic mean free path $l_{e}=12 \mu \mathrm{m}$. Figure (3) shows the experimental procedure to realize the electrostatic gate.
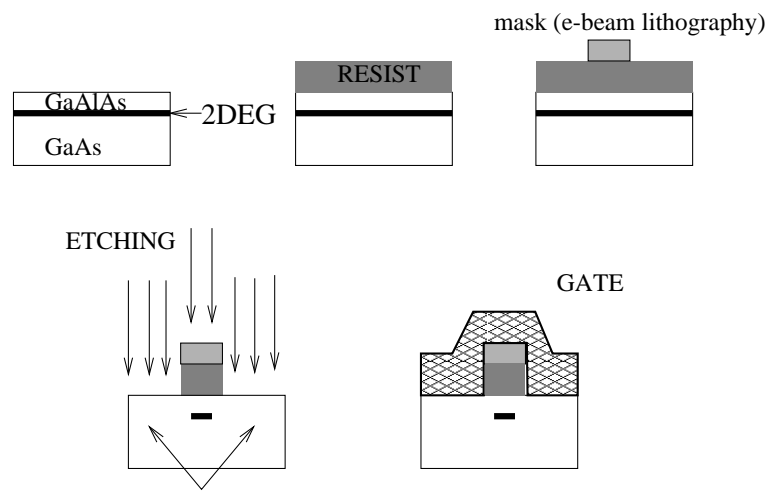

depletion of $2 \mathrm{DEG}$ induced by etching

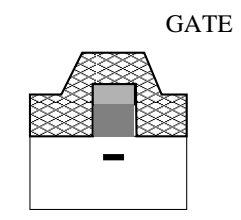

FIG. 3. Step by step process for the realisation of the gated samples.

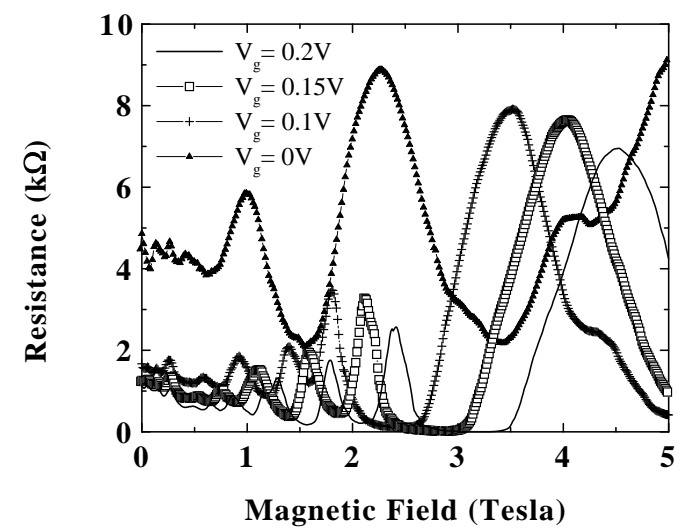

FIG. 4. Magnetoresistance of a $\mathcal{T}_{3}$ lattice versus the gate voltage showing Shubnikov-de-Haas oscillations.

A $200 \mathrm{~nm}$ thick insulator organic resist is first deposited on the top of the sample. We use then electron beam lithography and a lift-off technique to pattern the $\mathcal{T}_{3}$ lattice with an aluminium mask (see figure (11)). Reactive ion etching removes the resist from the unprotected regions. Then, etching using Argon ions transfers the pattern into the 2DEG. Due to the lateral depletion resulting from the etching process, the effective electrical width is considerably smaller than the wire width mask. The metal of the gate is then evaporated onto the whole surface. As a result, the gate is in intimate contact with the regions where the $2 \mathrm{DEG}$ has been destroyed whereas 
the thick resist layer considerably reduces the influence of the gate on the gas beneath the wires. As a consequence, using this approach, we reduce the gate action on the electron density and favour a pinching of the wires making the lattice. In figure (4), we present the magnetoresistance of the $\mathcal{T}_{3}$ network for several gate voltage values. The measurements are performed at $50 \mathrm{mK}$. From the Shubnikov-de-Haas oscillations we can extract the electron density versus the gate voltage.

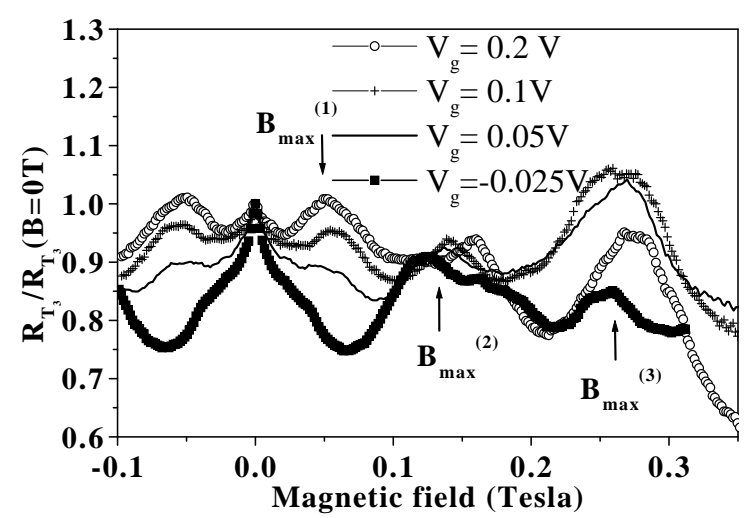

FIG. 5. Same as figure (4) but in the low magnetic field range dominated by the classical boundary scattering effects.

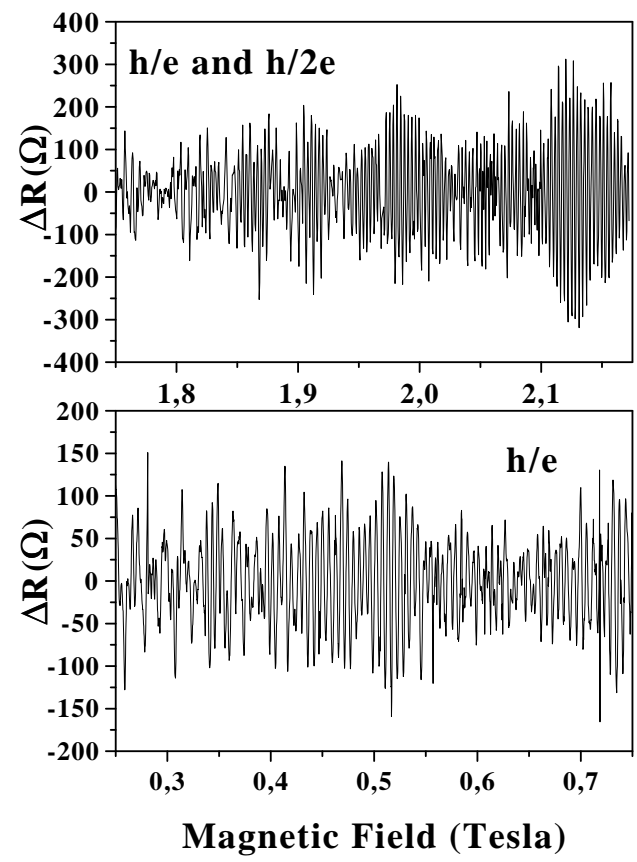

FIG. 6. $h / e$ and $h / 2 e$ oscillations after substracting the background.

In the low magnetic field regime we clearly observe large features which depend on the gate voltage (indicated by arrows in figure (5)). These features appear already at a temperature of $4 \mathrm{~K}$ and are almost temperature independent down to $50 \mathrm{mK}$, indicating a classical effect. In 2DEG systems, the mean free path at low temperature can be much larger than the width of the wire and the electrons are frequently scattered by the boundaries. Using a quasi-classical description, Ziman [8] considers the scattering of electrons by a rough boundary. The conclusions agree with our intuitive expectations : when the wavelength of the incident electron is much smaller than the average boundary roughness, the electron is strongly scattered in all directions. In the opposite case, when the Fermi wavelength is much larger, the incident electron undergoes specular reflections. These scatterings at the boundaries [9] lead naturally to the concept of the boundary scattering length, $l_{b}$, which is the average distance an electron can travel along a wire before a diffusive scattering event takes place. Then, the effective mean free path $l_{e f f}$ is such that $\frac{1}{l_{\text {eff }}}=\frac{1}{l_{\text {trans }}}+\frac{1}{l_{b}}$ and, as long as $l_{\text {trans }} \gg l_{b}$, boundary scattering dominates the momentum relaxation. The boundary scattering rate can be "tuned" using a magnetic field perpendicular to the wire. At zero or low magnetic field, electrons with a large component of momentum parallel to the boundaries contribute significantly to the conductivity. However, as the magnetic field increases, more and more electrons are forced to interact with the edges and random scattering reduces the effective mean free path $l_{e f f}$. Thus, the resistivity increases and subsequently saturates at some maximum value when the cyclotron radius is approximatively twice the wire width [9] :

$$
W=0.55 \times r_{c}\left(B_{\max }\right)=0.55 \times \frac{\sqrt{2 \pi \hbar^{2} n_{s}}}{e B_{\max }}
$$

Any further increase of the magnetic field will now lead to a drop of the resistivity since the probability for an electron to be backscattered by the edges is reduced. In figure (5) we observe 3 maxima corresponding to the 3 magnetic field values denoted $B_{\max }^{(1)}, B_{\max }^{(2)}$ and $B_{\max }^{(3)}$. These maxima correspond to three different widths we can identify in our samples : the width of the wires connecting the nodes, the diameter of the cavities formed at the sixfold coordinated and the threefold coordinated lattice sites. Using equation (11) we can deduce the real dimension of the electron path. The channel number can be deduced from the comparison between the Fermi wavelength and the wire width $\left(N=\frac{W^{(3)}}{\lambda_{F} / 2}\right)$. These results are reported Table IV.

The identification of $W^{(3)}$ to the wire width gives reasonable numerical values. Nevertheless, the values obtained for the cavities diameters are overestimated, which is not surprising since this wire model is not really adapted to the particular shape of the junctions.

The unexpected frequency doubling leads us to define 
two magnetic field regimes : a low field regime (between $0.4 \mathrm{~T}$ and $1.2 \mathrm{~T}$ ) where we observe only $h / e$ oscillations and a high field regime (from $1.2 \mathrm{~T}$ to $2 \mathrm{~T}$ ) where the $h / 2 e$ oscillations appear in addition to the $h / e$ ones. We substract a polynomial fit of the global magnetoresistance to the experimental points in order to extract the oscillations. This resulting signal (see figure (6) ) is then Fourier transformed.

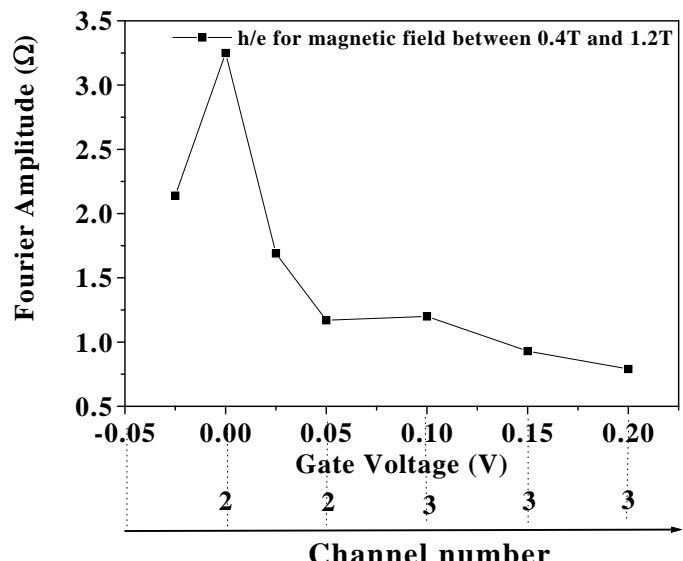

FIG. 7. Variation of the $h / e$ peak amplitude of the Fourier transform versus the channel number for the low magnetic field regime.

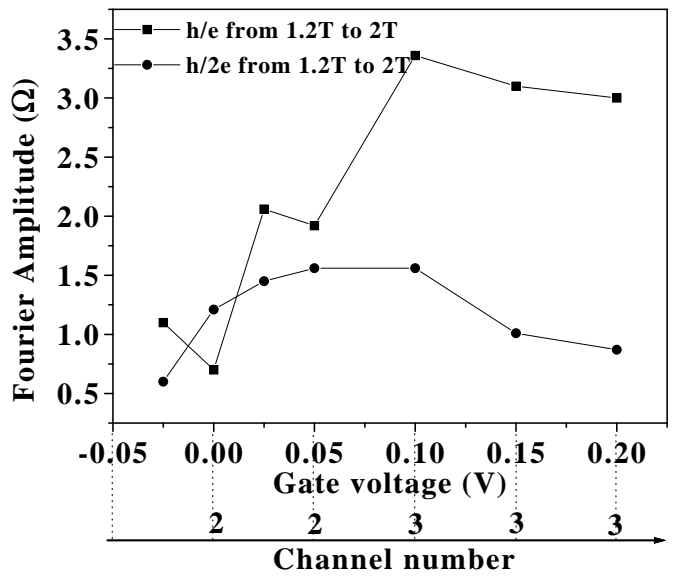

FIG. 8. Variation of the $h / e$ and $h / 2 e$ peak amplitudes of the Fourier transform versus the channel number for the high magnetic field regime.

Let us now discuss the gate voltage influence on the oscillations in both regimes which is reported in figures (7) and (8), where we have plotted the Fourier transform peak magnitude against the channel number. In the low field regime the $\mathrm{AB}$ amplitude clearly increases with decreasing the electrical wire width. This is in agreement with the work of M. Büttiker et al. on a simple loop [10]. Essentially, their calculation shows that if $N$ is the channel number, the $h / e$ signal results from $N^{2}$ uncorre- lated contributions whose stochastic averaging gives rise to a decrease of the AB oscillation amplitude with $N$. Note that in our experiments the amplitude of the Fourier transform falls down at a critical gate voltage (about $0 \mathrm{~V}$ corresponding to a channel number close to 2). This may be due to the wire pinch-off induced by the gate. As a result, the cutting of some wires supresses the cage effect and consequently reduces the $h / e$ signal. In the high field regime we observe a different behavior between the $h / e$ and the $h / 2 e$ periods. The $h / e$ amplitude decreases monotonously with the reduction of the channel number. On the other hand, the $h / 2 e$ oscillations seem to have a constant magnitude versus the gate voltage. This fact suggests a different mechanism to explain the two periodicities in this high field regime. More surprisingly, the $h / e$ signal itself behaves in an opposite manner in the two regimes : whereas, as discussed above, the $\mathrm{AB}$ amplitude at low field has the expected channel number dependence, in the high field regime the $h / e$ signal increases with $N$. This leads us to raise the hypothesis that we are dealing with different physical phenomena in the two magnetic field regimes. Up to now the origin of the $h / 2 e$ peak at high magnetic fields and, more, the field strength effect on the $h / e$ peak amplitude, remain unclear.

\section{THE EFFECT OF DISORDER}

In this section, we discuss the influence of an elastic disorder on the periodicity of the $\mathrm{AB}$ oscillations in the $\mathcal{T}_{3}$ lattice. In order to simplify this analysis, we consider that there exists only one conduction channel. Of course this hypothesis is somehow unrealistic but it allows us to get a partial answer to this problem. The technical details can be found in ref. [11].

From a theoretical point of view, there are several ways to introduce disorder in a system. For instance, one can consider a random scattering matrix on each bond of the network or at each node. We can also put some additionnal dead-end bonds of random lengths to mimick the dephasing due to impurities. Here, we adopt another point of view by changing the length of each quantum path. One way to do this practically, is to make a random modulation $\Delta l$ of each bond of length $l$ without modifying the phase factor due to the magnetic field. Indeed, since each bond is considered as strictly one-dimensional, the circulation of the vector potential along one link is not affected by reflections of the wavepacket on this link. Thus, in the Landauer-like transmission formalism that we have used, we only include these length fluctuations in the quantity $k l$ which is the main parameter of our study [11]. In the following, we focus on the transmission properties of a piece of the $\mathcal{T}_{3}$ lattice and we compare it to a piece of the square lattice (see figure (9)). 

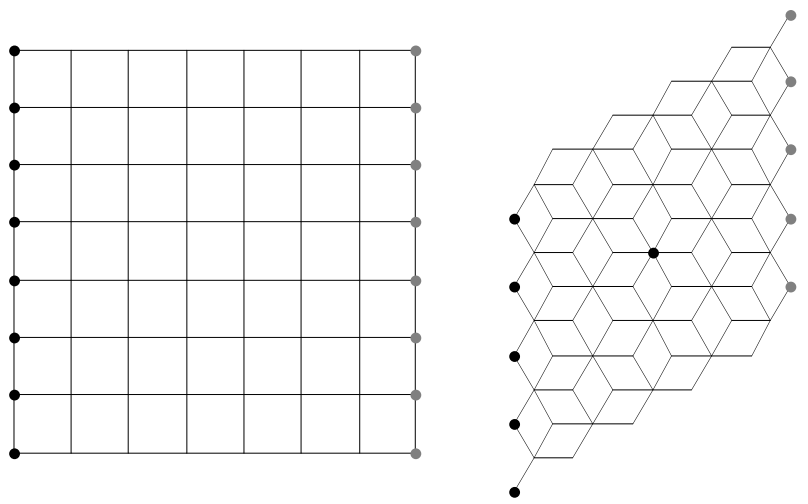

FIG. 9. A piece of the square lattice (left) and of the $\mathcal{T}_{3}$ network (right). Black (respectively grey) dots represent the connections to the input (respectively output) channels. The central black dot is the input channel chosen for the bulk injection.

In the pure case $(\Delta l=0)$ the transmission coefficient $T$ depends on the wave vector $k$ of the incoming wave function and on the reduced flux $f$. We have displayed in figure (10), the averaged transmission coefficient for $k l \in[0,2 \pi]$. One clearly observes some peaks for rationnal $f$ (extended Bloch-like eigenstates) reminiscent of the butterfly-like structure of the energy spectrum. Due to the existence of the $\mathrm{AB}$ cages, the transmission coefficient is minimum at $f=1 / 2$ for the $\mathcal{T}_{3}$ network but it does not strictly vanish. This is due to the existence of dispersive edges states [12] that are able to carry current even for $f=1 / 2$. Nevertheless, when one injects the current in the bulk of the sample, we obtain $T=0$ for $f=1 / 2$ as expected. Note that for both systems, the signal is anharmonic but is periodic with period $\phi_{0}$ leading to a $h / e$ periodicity for the magneto-resistance.

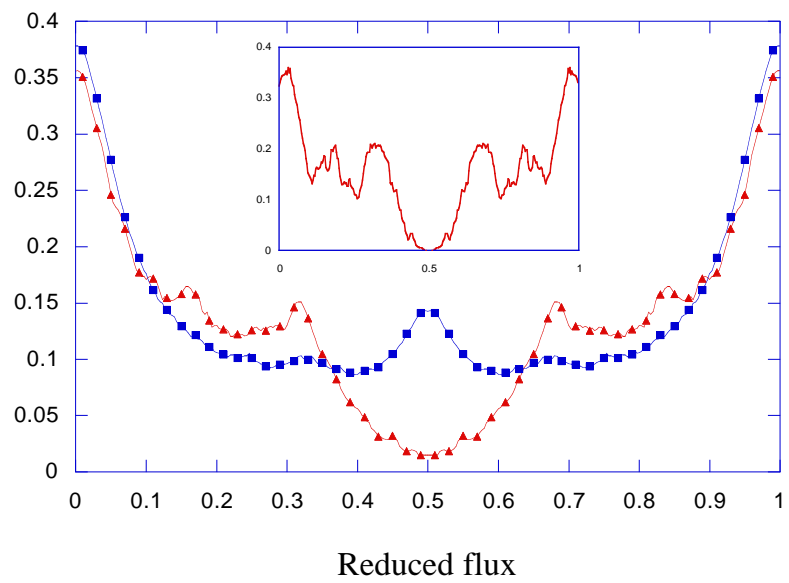

FIG. 10. Averaged transmission coefficient as a function of the reduced flux for the square lattice (square) and for the $\mathcal{T}_{3}$ network (triangle). Inset : averaged transmission coefficient for the $\mathcal{T}_{3}$ network with a single input channel in the bulk of the network.

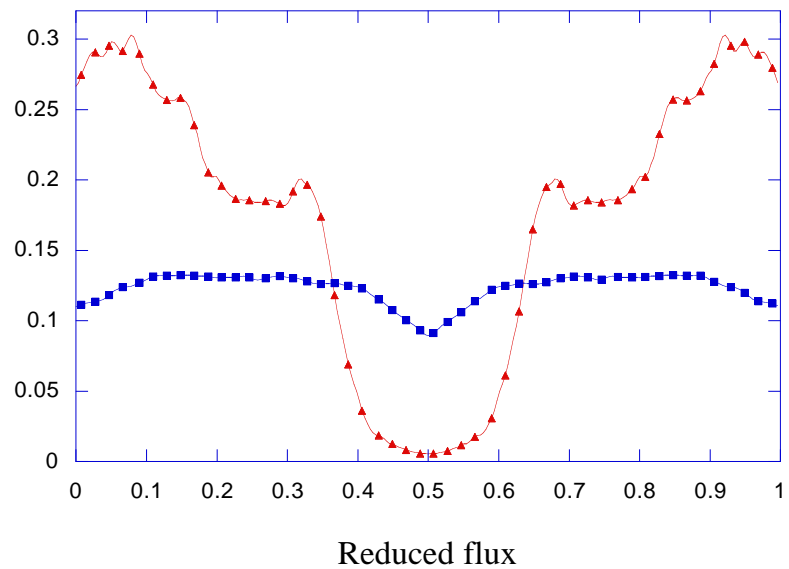

FIG. 11. Transmission coefficient averaged over 50 configurations of disorder for $k l=\pi / 3$ and $k \Delta l=1.47$ as a function of the reduced flux.

The main question is to know how this periodicity is modified when we introduce the disorder. It is clear that if the disorder is very strong $(k \Delta l \simeq \pi)$, there is no phase coherent transmission and we expect a vanishing transmission after averaging over disorder. Consequently, there might be interesting phenomenon for weak enough disorder. We emphasize that the notion of weak and strong disorder is related to the product $k \Delta l$ and thus depends on $k$. Note that this way to model a static disorder is not expected to capture all the features of experimental data. For instance, it yields an effective disorder which increases with the Fermi energy, but this effect could well be completely compensated by the energy dependence of the impurity scattering cross section in real systems. We have shown in figure (11) the effect of disorder on the transmission coefficient as a function of the magnetic flux. It is clearly seen that for the square network, the periodicity is no longer $\phi_{0}$ but $\phi_{0} / 2$. This new periodicity is simply due to phase coherent pairs of timereversed trajectories according to the weak-localization picture. The most striking feature is that for the $\mathcal{T}_{3}$ lattice, the transmission coefficient remains $\phi_{0}$-periodic with a large amplitude. This strongly suggests that the cage effect (which locks the phase of the oscillations) survives for this strength of disorder. Physically, it means that a strong dephasing is required to destroyd the completely destructive quantum interferences responsible of the $\mathrm{AB}$ cages. In addition, note that, for this energy, the signal of the $\mathcal{T}_{3}$ lattice transmission coefficient is about 10 times larger than the one of the square network. In ref. [12] we report on other energy of the incoming wavepacket where we find always the same type of feature.

\section{CONCLUSIONS}

Localisation phenomenon induced by the magnetic field on the $\mathcal{T}_{3}$ topology has been confirmed in the 
GaAlAs/GaAs system. The frequency doubling in the high magnetic field regim is systematically observed and displays a different behavior versus the channel number compared to the fundamental period. The theoretical predictions of the effect of disorder motivate the investigation of such networks in metallic systems.

[1] D. R. Hofstadter, Phys. Rev. B14, 2239 (1976).

[2] J. Vidal, R. Mosseri, and B. Douçot, Phys. Rev. Lett.81, 5888 (1998).

[3] C. C. Abilio, P. Butaud, Th. Fournier, and B. Pannetier, J. Vidal, S. Tedesco and B. Dalzotto, Phys. Rev. Lett.83, 5102 (1999).

[4] E. Serret et al., in preparation.

[5] C. Naud, G. Faini, and D. Mailly, Phys. Rev. Lett.86, 5104 (2001).

[6] F. P. Milliken, S. Wasburn, C. P. Umbach, R. B. Laibowitz, and R. A. Webb, Phys. Rev. B36, 4465 (1987).

[7] T. J. Thornton, M. Pepper, H. Ahmed, D. Andrews, and G. J. Davies, Phys. Rev. Lett.56, 1198 (1986).

[8] J. M. Ziman, Electrons and phonons Chap. 11, Oxford Univ. Press UK (1960).

[9] T. J. Thornton, M. L. Roukes, A. Scherer, and B. P. E. Van der Gaag, Coherence in Mesoscopic Systems, edited by B. Kramer, Plenum Press (New York and London), series B : Physics Vol. 254, 153 (1990).

[10] M. Büttiker, Y. Imry, R. Landauer, and S. Spinhas, Phys. Rev. B31, 6207 (1985).

[11] J. Vidal, G. Montambaux, and B. Douçot, Phys. Rev. B62, R16294 (2000).

[12] J. Vidal, P. Butaud, B. Douçot, and R. Mosseri, to be published in Phys. Rev. B.

Table I :

\begin{tabular}{lllllllll}
$\begin{array}{l}\text { Gate } \\
\text { voltage }\end{array}$ & $\begin{array}{l}n_{s} \\
10^{11} \mathrm{~cm}^{-2}\end{array}$ & $\begin{array}{l}B_{\max }^{(1)} \\
\text { (Tesla) }\end{array}$ & $\begin{array}{l}W^{(1)} \\
(\mu m)\end{array}$ & $\begin{array}{l}B_{\max }^{(2)} \\
(\text { Tesla })\end{array}$ & $\begin{array}{l}W^{(2)} \\
(\mu m)\end{array}$ & $\begin{array}{l}B_{\max }^{(3)} \\
(\text { Tesla })\end{array}$ & $\begin{array}{l}W^{(3)} \\
(\mu m)\end{array}$ \\
\hline$\overline{V_{g}=0.2 V}$ & 1.48 & 0.052 & 0.67 & 0.159 & 0.219 & 0.274 & 0.1273 \\
$V_{g}=0.15 V$ & 1.32 & 0.052 & 0.63 & 0.154 & 0.219 & 0.268 & 0.123 & 3 \\
$V_{g}=0.1 V$ & 1.17 & 0.056 & 0.56 & 0.140 & 0.221 & 0.262 & 0.1183 \\
$V_{g}=0.05 V$ & 0.9 & hard to locate & 0.137 & 0.199 & 0.270 & 0.1012 \\
$V_{g}=0 V$ & 0.83 & hard to locate & 0.122 & 0.213 & 0.256 & 0.1012 \\
\hline \hline
\end{tabular}

